\title{
BORDISM OF DIFFEOMORPHISMS
}

BY M. KRECK

Communicated by P. T. Church, April 28, 1976

1. Introduction. In this note we determine the bordism groups $\Delta_{n}$ of orientation preserving diffeomorphisms of $n$-dimensional closed oriented smooth manifolds. These groups were introduced by W. Browder [1]. Winkelnkemper showed that each diffeomorphism of the sphere $S^{n}$ is nullbordant [7]. On the other hand, he showed that $\Delta_{4 k+2}$ is not finitely generated. Medrano generalized this result to $\Delta_{4 k}$ [5]. For this he introduced a powerful invariant in the Witt group $W_{ \pm}(\mathbf{Z}, \mathbf{Z})\left(I_{ \pm}\right.$in Medrano's notation) of isometries of free finite-dimensional Z-modules with a symmetric (antisymmetric) unimodular bilinear form. The invariant is given by the middle homology modulo torsion, the intersection form and the isometry induced by the diffeomorphism. For a diffeomorphism $f: M \rightarrow M$ we denote this invariant by $I(M, f)$, the isometric structure of $(M, f)$. It is a bordism invariant and leads to a homomorphism $I: \Delta_{2 k} \rightarrow$ $W_{(-1)^{k}}(\mathbf{Z}, \mathbf{Z})$.

Neumann has shown that the homomorphism $I$ is surjective, that $W_{ \pm}(\mathbf{Z}, \mathbf{Z})$ $\otimes \mathbf{Q} \cong \mathbf{Q}^{\infty}$ and that $W_{ \pm}(\mathbf{Z}, \mathbf{Z})$ contains infinitely many summands of orders 2 and $4[6]$. On the other hand, $W_{ \pm}(\mathbf{Z}, \mathbf{Z})$ is a subgroup of $W_{ \pm}(\mathbf{Z}, \mathbf{Q})$, the Witt group of isometries of finite-dimensional $\mathbf{Q}$-vector spaces. This group plays an important role in the computation of bordism groups $C_{2 k-1}$ of odd-dimensional knots, which can be embedded in $W_{(-1)^{k}}(\mathbf{Z}, \mathbf{Q})$. It is known that $W_{ \pm}(\mathbf{Z}, \mathbf{Q}) \cong$ $\mathbf{Z}^{\infty} \oplus \mathbf{Z}_{2}^{\infty} \oplus \mathbf{Z}_{4}^{\infty}[3]$. Thus the group $W_{ \pm}(\mathbf{Z}, \mathbf{Z})$ is also of the form $\mathbf{Z}^{\infty} \oplus \mathbf{Z}_{2}^{\infty} \oplus$ $\mathbf{Z}_{4}^{\infty}$.

It turns out that the isometric structure is essentially the only invariant for bordism of diffeomorphisms.

2. Bordism of odd-dimensional diffeomorphisms. Two diffeomorphisms $\left(M_{1}, f_{1}\right)$ and $\left(M_{2}, f_{2}\right)$ are called bordant if there is a diffeomorphism $(N, F)$ on an oriented manifold with boundary such that $\partial(N, F)=\left(M_{1}, f_{1}\right)+\left(-M_{2}, f_{2}\right)$. The bordism classes $\left[M^{n}, f\right]$ form a group under disjoint sum, called $\Delta_{n}$.

The mapping torus of a diffeomorphism $(M, f)$ is $M_{f}=I \times M /(0, x) \sim$ $(1, f(x))$. This construction leads to a homomorphism $\Delta_{n} \rightarrow \Omega_{n+1}([M, f] \mapsto$ $\left[M_{f}\right]$ ), where $\Omega_{n+1}$ is the ordinary bordism group of oriented manifolds.

In [4] we proved the following result.

AMS (MOS) subject classifications (1970). Primary 57D90. 
THEOREM 1. For $k>2$ the map $[M, f] \mapsto\left([M],\left[M_{f}\right]\right)$ is an isomorphism $\Delta_{2 k-1} \rightarrow \Omega_{2 k-1} \oplus \hat{\Omega}_{2 k}$, where $\hat{\Omega}_{2 k}$ is the kernel of the signature homomorphism $\tau$.

3. The even-dimensional case. Consider triples $(G,\langle\rangle, h$,$) , where G$ is a finite-dimensional free $Z$-module, $\langle$,$\rangle a symmetric (resp. antisymmetric) unimod-$ ular bilinear form on $G$ and $h$ an isometry of $(G,\langle\rangle$,$) . (G,\langle\rangle, h$,$) is called hy-$ perbolic if there exists an invariant subkernel, i.e. a subspace $U \subset G$ with $U \subset$ $U^{\perp}, 2 \operatorname{dim} U=\operatorname{dim} G$ and $h(U) \subset U .(G,\langle\rangle, h$,$) and \left(G^{\prime},\langle,\rangle^{\prime}, h^{\prime}\right)$ are called bordant if $(G,\langle\rangle, h,) \oplus\left(G^{\prime},-\langle,\rangle^{\prime}, h^{\prime}\right)$ is hyperbolic. This is an equivalence relation. The equivalence classes form a group under orthogonal sum, called $W_{+}(\mathbf{Z}, \mathbf{Z})$ (resp. $W_{-}(\mathbf{Z}, \mathbf{Z})$ ).

The isometric structure of a diffeomorphism $\left(M^{2 k}, f\right)$ is given by $\left(H_{k}(M ; \mathbf{Z}) /\right.$ Tor, $\left.{ }^{\circ}, f_{*}\right)$, where $\circ$ is the intersection form. If $(M, f)$ bounds a diffeomorphism $(N, F)$ the isometric structure is hyperbolic, an invariant subkernel being given by the kernel of $i_{*}: H_{k}(M ; \mathbf{Z}) /$ Tor $\rightarrow H_{k}(N ; \mathbf{Z}) /$ Tor, so we have a homomorphism $I: \Delta_{2 k} \rightarrow W_{(-1) k}(\mathbf{Z}, \mathbf{Z})$. Neumann has shown that this homomorphism is surjective [6].

THEOREM 2. For $k>1$ the homomorphism

$$
\begin{aligned}
& \Delta_{2 k} \rightarrow W_{(-1)^{k}}(\mathrm{Z}, \mathrm{Z}) \oplus \hat{\Omega}_{2 k} \oplus \Omega_{2 k+1}, \\
& {[M, f] \mapsto\left(I(M, f),[M]-\tau(M)\left[P_{k} \mathbf{C}\right],\left[M_{f}\right]\right)}
\end{aligned}
$$

is an isomorphism ( $k$ even), injective with cokernel $\mathbf{Z}_{2}(k$ odd $)$.

4. Idea of the proof. Consider a diffeomorphism $\left(M^{n}, f\right)$ such that $M$ and $M_{f}$ are nullbordant. This implies that there exists a differentiable map $g$ : $N^{n+2} \rightarrow S^{1}$ with $\partial N=M_{f}$ and $\left.g\right|_{\partial N}$ the canonical projection from $M_{f}$ to $S^{1}$. Let $x \in S^{1}$ be a regular value of $g . F:=g^{-1}(x)$ is a 1 -codimensional submanifold of $N$ with trivial normal bundle meeting $\partial N$ transversally along $\partial F$ : Cut $N$ along $F$ to obtain a differentiable manifold $N_{F}$ with corners. The boundary of $N_{F}$ consists of two copies $F_{0}$ and $F_{1}$ of $F$ with opposite orientations and a manifold $V=\partial N_{\partial F}$ fibred over the unit interval $I . \partial V=\partial F_{0}+\partial F_{1}$. The corners of $N_{F}$ are at $\partial F_{0}$ and $\partial F_{1}$.

We now make the following strong assumption (compare [2, 2.3]): The components of $F$ are simply connected and $F_{0}$ and $F_{1}$ are deformation retracts of $N_{F}$.

Then $\left(N_{F} ; F_{0}, F_{1}\right)$ is a relative $h$-cobordism between $\left(F_{0}, \partial F_{0}\right)$ and $\left(F_{1}, \partial F_{1}\right)$. For $n>5$ the $h$-cobordism theorem implies that the diffeomorphism on $M$ can be extended to a diffeomorphism of $F$.

Our aim is, starting with an arbitrary $N_{F}$ to obtain an $N_{F}^{\prime}$, which satisfies the above assumption. For this we modify $N_{F}$ by addition and subtraction of handles. If $n$ is odd $(n \neq 3)$ we have shown in [4] that this works. If $n$ is even 
$(n>2)$ and $I(M, f)=0$ we can do the same. The details of the proof will appear elsewhere.

\section{REFERENCES}

1. W. Browder, Surgery and the theory of differentiable transformation groups, Proc. Conf. on Transformation Groups (New Orleans, La., 1967), Springer-Verlag, New York, 1968, pp. 1-46. MR 41 \#6242.

2. W. Browder and J. Levine, Fibering manifolds over a circle, Comment. Math. Helv. 40 (1966), 153-160. MR 33 \#3309.

3. M. A. Kervaire, Knot cobordism in codimension two, Manifolds-Amsterdan 1970 (Proc. Nuffic Summer School), Lecture Notes in Math., vol. 197, Springer-Verlag, Berlin and New York, 1971, pp. 83-105. MR 44 \#1016.

4. M. Kreck, Cobordism of odd-dimensional diffeomorphisms, Topology (to appear).

5. S. López de Medrano, Cobordism of diffeomorphisms of $(k-1)$-connected $2 k$ manifolds, Proc. Second Conf. on Compact Transformation Groups (Amherst, 1971), Part I., Lecture Notes in Math., vol. 298, Springer-Verlag, Berlin and New York, 1972, pp. 217227. MR 51 \#1847.

6. W. D. Neumann, Signature related invariants of manifolds (in preparation).

7. H. E. Winkelnkemper, On equators of manifolds and the actions of $\theta^{n}$, Thesis, Princeton Univ., 1970.

MATHEMATISCHES INSTITUT DER UNIVERSITÄT, SONDERFORSCHUNGSBEREICH THEORETISCHE MATHEMATIK, WEGELERSTR. 10,53 BONN, GERMANY 